\title{
Modeling and Control of Ankle Actuation Platform for Human-Robot Interaction
}

\author{
Ata Otaran and Ildar Farkhatdinov \\ School of Electronic Engineering and Computer Science, \\ Queen Mary University of London, UK \\ $\{$ a.otaran, i.farkhatdinov\}@qmul.ac.uk
}

\begin{abstract}
We present the design of a one-degree-of-freedom ankle actuation platform for human-robot interaction. The platform is actuated with a DC motor through a capstan drive mechanism. The results for platform dynamics identification including friction characterisation are presented. Control experiments demonstrate that a linear regulator with gravity compensation can be used to control the inclination of the platform efficiently.
\end{abstract}

Keywords: haptic interfaces $\cdot$ lower limb $\cdot$ control

\section{Introduction}

Most of the haptic devices used in telerobotics or virtual reality (VR) are used to apply force or tactile feedback on a user's upper limbs. However, in many haptic applications, providing feedback to the lower limb can be useful and efficient. In this paper, we describe a robotic interface to provide force feedback through ankle actuation. There have been various ankle actuation platforms developed previously for neuromotor rehabilitation, as ankle health is crucial for the mobility of individuals. Normally robotic ankle rehabilitation devices comprise several degrees-of-freedom (DoF). A widely known rehabilitation system, Rutgers Ankle [6], used a Stewart platform equipped with a single force sensor under the foot. Reducing the mechanics of the Stewart platform to 3-DoF versions led to the development of a 3SPS/S and a 3-RSS/S type parallel mechanisms [2] and in [9] respectively. An Agile Eye type 3-DOF spherical parallel mechanism was used in [10]. In [11] and [12], authors presented impedance type rehabilitation devices for characterizing the ankle impedance to obtain valuable insight into post-stroke recovery in patients. Assist-On Ankle [3] made use of Bowden cable based series elastic actuated parallel mechanism that can align with the subject ankle axis and deliver plantar flexion/dorsiflexion and pronation/supination exercises. As can be inferred from the examples parallel mechanisms are ideal for ankle joint actuation as the addition of DoF does not result in a vastly increased device inertia due to having grounded motors and the ankle joint does not require a large workspace. Relatively simpler ankle tilt platforms were also used in VR based studies like serious games [7] for rehabilitation or studying human postural control [1]. 
In this paper, we present the development of an ankle actuation interface for human-robot interaction research. In particular, we plan to use the proposed interface for emulation of movement sensation in virtual reality and telerobotics applications. As the single DOF, we selected plantar flexion and dorsiflexion movements as forwards and backwards is the primary axis of movement used in daily life. We decided to employ an impedance type interface with a backdriveable actuator that can also apply high enough ankle torque. Addition of a compliant force sensor would decrease the available control bandwidth. Using high quality force sensing will increase the cost of the device. Although it is not desirable to apply large amplitude and high frequency signals to the users to avoid human reflexes, we would like to be able to apply high frequency and low amplitude feedback to be able to account for smaller bumps in the terrain. Ability to apply feedback in a wide range of amplitudes and frequencies not only helps display a variety of feedback but it may also help superimpose different types of information in the feedback such as guidance and performance indication purposes. In order to single out forces applied by a human, a disturbance observer based force estimation method will be utilized [8].

This paper elaborates on the design and modeling stages that come before the implementation of the envisioned reaction torque observer implementation. Section 2 explains design of the proposed interface. A dynamic model including inertia and friction characterization and preliminary control performance evaluation of the device are provided in section 3 which describes gravity compensation, angular orientation control, and friction compensation. Section 4 summarizes the project and discusses potential future improvements.

\section{Mechanical design description}

This section explains how mechanical components are put together for the construction of the initial iteration of the experiment setup. Figure 1 shows the prototype designed to be used in the initial experiments. The device is composed of four main mechanical assemblies: the base, the tilting platform, the motor and the capstan transmission. These main parts are explained in detail below.

The base supports the stability of the whole system and constrains the motion of the platform to one revolute DoF. The lower part of the base is made of aluminum sigma profiles. Sigma profiles are arranged to cover a large area for the stability of the base during operation. The vertical stands, made of $10 \mathrm{~mm}$ thick aluminum are attached to the base to support the rotation of the tilt platform.

The tilt platform is composed of two shafts, two quarter circular aluminum pieces (pulleys), four pieces of aluminum sigma profiles, a wooden plate, capstan mounting units and basic mechanical connection parts. The shafts and double bearings on the base provide the necessary constraint for a single DoF rotational motion around the ankle axes. Pulleys connect the shafts with the plate and are actuated by the motor using capstan transmission. 


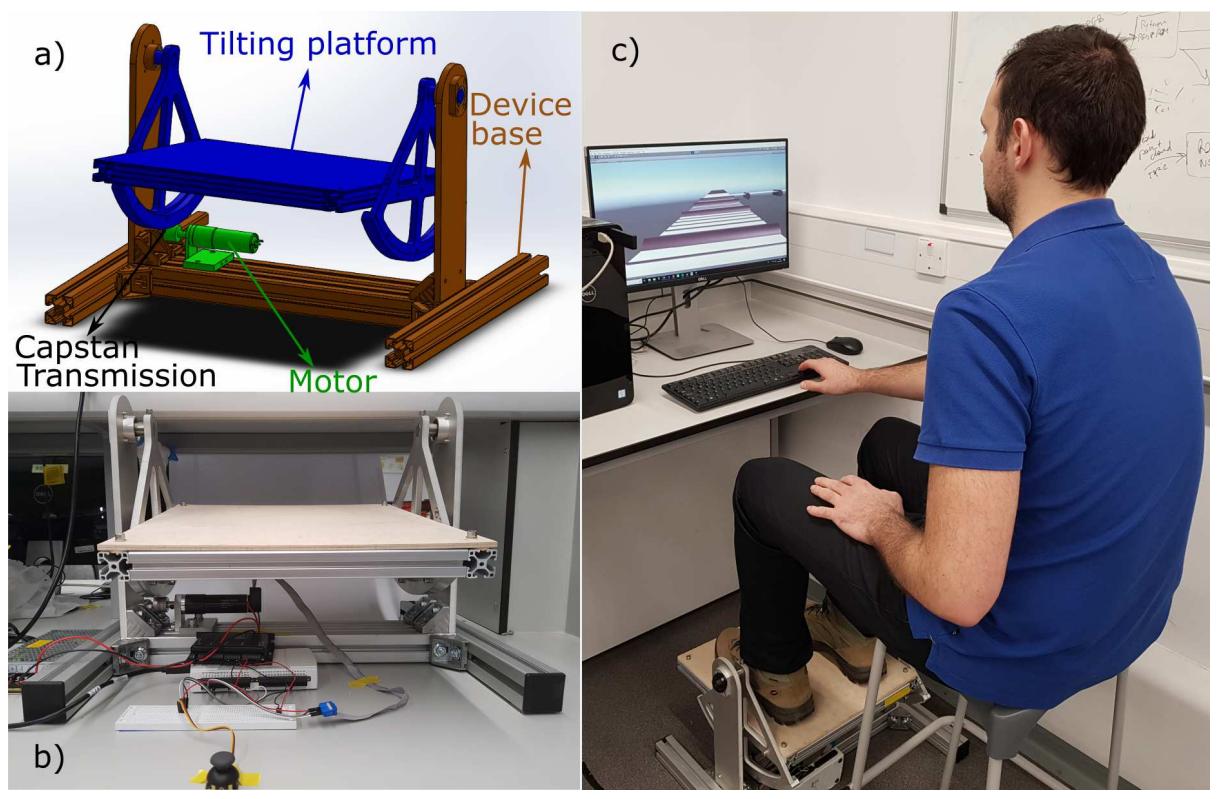

Fig. 1. a) A computer aided design image of the device b) image of the device along with the electronic components after construction c) image of a user utilizing the device with a sample VR application instance.

Capstan drive, is used to transmit the motion of the motor to the tilt platform. One end of the capstan cable is mounted on the side of the pulley and the other one is mounted underneath one of the sigma profiles of the plate assembly. The latter end of the cable is connected to a spring which can alleviate a sudden rise of tensile stress on the cable. The spring is chosen to be stiff enough to avoid introducing additional vibration in the working bandwidth of the device. An additional sliding mechanism was installed to control the cable tension.

The motor assembly is composed of a geared DC motor with encoder (Maxon RE30, gear ratio 1:14), aluminium attachment elements fixed to the base and the driving pinion. The capstan is revolved around the pinion 5 times in order to avoid slipping under high torques. The properties of the motor and the gear are selected such that high enough torques at the ankle can be generated and, at the same time, the device is backdrivable enough for reliable impedance type operation.

\section{Modeling and control}

In order to achieve a high operation performance with the platform, its dynamics were modeled and relevant control was developed. Figure 2 presents the dynamic model of the system which includes the DC-motor with gear, the cable transmission and the tilting platform. The parameters of the model are described in 
Table 1 and many of them can be obtained from the DC-motor's datasheet and CAD design of the platform.

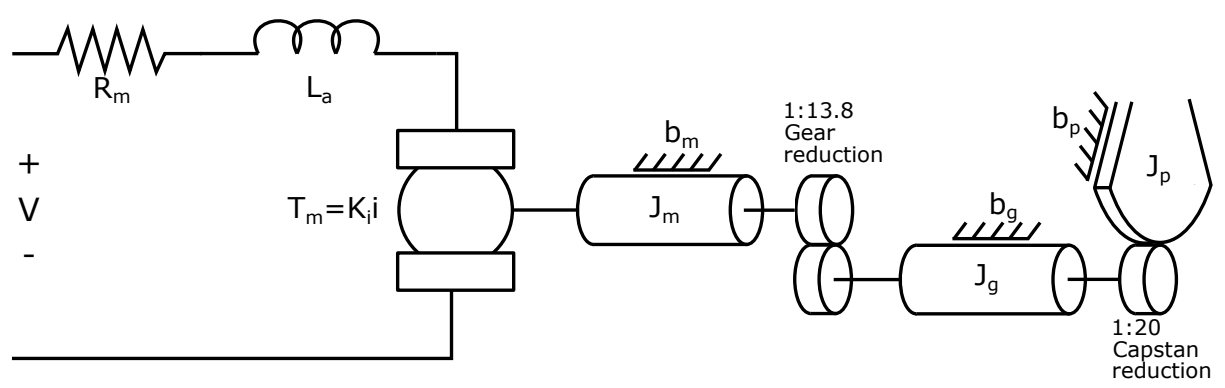

Fig. 2. Dynamic model of the bodies and transmission systems involved in the ankle tilt platform

Table 1. Ankle platform characteristic parameters

\begin{tabular}{l|r|l}
\hline \hline$J_{a}-$ inertia of the motor & 33.5 & $\mathrm{gr}-\mathrm{cm}^{2}$ \\
$J_{g}-$ inertia of the gearhead & 0.8 & $\mathrm{gr}^{2} \mathrm{~cm}^{2}$ \\
$J_{p}-$ inertia of the ankle platform & 0.0717 & $\mathrm{~kg}-\mathrm{m}^{2}$ \\
$J_{e q}-$ equivalent inertia at motor end & 42.92 & $\mathrm{~g}^{2} \mathrm{~cm}^{2}$ \\
$r_{g}-$ gearhead reduction ratio & $13.8: 1$ & \\
$r_{c}-$ capstan reduction ratio & $20: 1$ & \\
$L-$ motor inductance & 0.119 & $\mathrm{mH}$ \\
$R-$ motor resistance & 0.611 & $\mathrm{Ohm}$ \\
$K_{i}-$ motor torque constant & 25.9 & $\mathrm{mN}-\mathrm{m} / \mathrm{A}$ \\
$K_{b}-$ motor speed constant & 369 & $\mathrm{rpm} / \mathrm{V}$ \\
$\tau_{m}-$ mechanical time constant & 3.05 & $\mathrm{~ms}$ \\
$T_{m}-$ motor nominal torque & 85.6 & $\mathrm{mN}-\mathrm{m}$ \\
$w_{m}-$ motor nominal speed & 8050 & $\mathrm{rpm}$ \\
$T_{a n k l e}-$ ankle axis nominal torque & 23.6 & $\mathrm{~N}-\mathrm{m}$ \\
$w_{\text {ankle }}-$ ankle axis nominal speed & 175 & $\mathrm{r} / \mathrm{s}$ \\
$\theta_{\text {lim }}-$ workspace limits & \pm 43 & $o$ \\
\hline
\end{tabular}

We describe the dynamics with the following transfer functions. The control voltage $V(S)$ (reference voltage for current control) to angular orientation of the platform, $\theta_{m}(s)$ transfer function is described as follows:

$$
\frac{s \theta_{m}(s)}{V(s)}=\frac{K_{i} / R}{J_{e q} s+b_{e q}}
$$


where $J_{e q}=J_{m}+J_{g} / r_{g}^{2}+J_{p} /\left(r_{g} r_{c}\right)^{2}$ and $b_{e q}=K_{i} K_{b} / R+b_{m}+b_{g} / r_{g}+b_{c} / r_{g} r_{c}$ (see Table 1 for notation). Some of the parameters, such as damping, should be estimated through experimental methods such as motor spin down and observation of pendulum oscillation dissipation tests. The transfer function from a human ankle torque input, $T_{\text {ankle }}(s)$, to angular orientation of the platform, $\theta_{m}(s)$, is

$$
\frac{s \theta_{m}(s)}{T_{\text {ankle }}(s)}=\frac{-1 /\left(r_{g} r_{c}\right)}{J s+b}
$$

The designed platform can be modelled as a linear system and therefore we utilize a PID regulator with gravity compensation to control the angular orientation of the ankle platform. Initially, the parameters of the PID regulator were selected based on the linear model of the system to achieve a critically damped response without taking into account the user's ankle dynamics. Since user's feet will be a big consideration in the selection of the parameters, a more reliable model-based feedback control scheme will be developed in parallel to the performance evaluation trials with different users.

\subsection{Gravity compensation}

Since the centre of gravity of the system is not aligned with the axis of rotation the gravity compensation control was introduced in addition to the PID regulator. For the gravity compensation a simplified model of the device was used as shown in Figure 3. The location of the COM was retrieved from the CAD model.

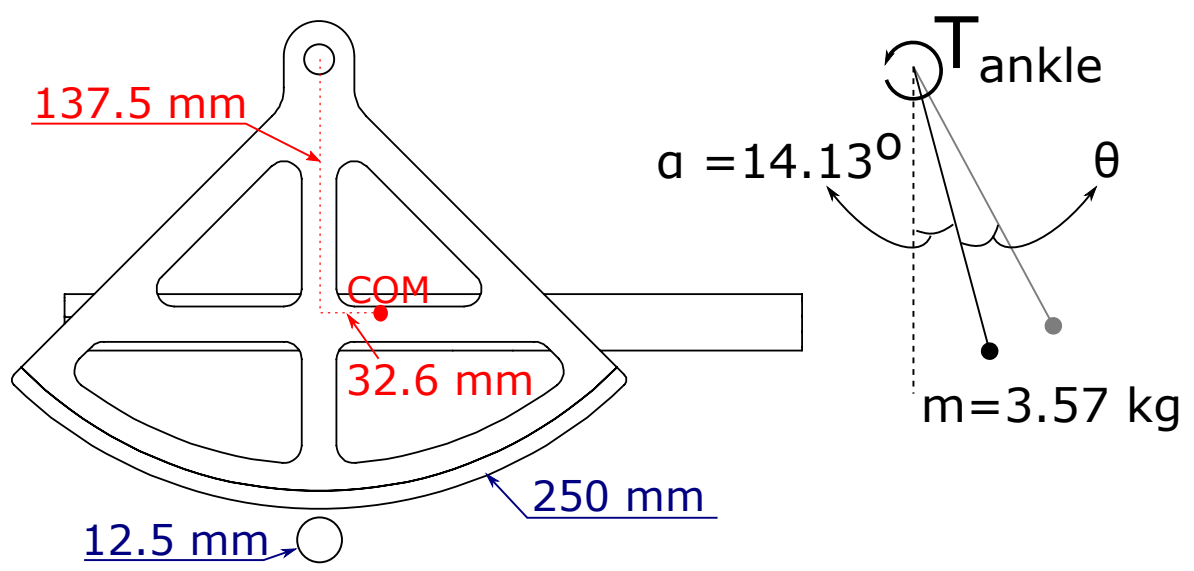

Fig. 3. Simplified model of the device. Due to the extra space in the front the center of mass is shifted towards the longer side of the plate. 
To compensate for gravity, the torques induced around the axis of revolution due to the load should be provided by the actuator in a feed-forward manner. Equation 3 is the required calculation for the feedforward gravity compensation signal:

$$
T_{\text {gravity }}=m g l \sin (\theta+\alpha)
$$

where $\theta$ is the tilt angle of the plate and $\alpha$ is the angle between the axis that is orthogonal to the plate and the shortest line from the COM to the axis of rotation as seen in Figure 3. The DC-motor current required for gravity compensation is calculated as:

$$
i_{m}=\frac{m g l \sin (\theta+\alpha)}{K_{i} r_{g} r_{c}}
$$

The general system equation can be rewritten as:

$$
\begin{aligned}
T_{\text {motor }}+T_{\text {human }} & =J_{e q} \ddot{\theta}+b_{e q} \dot{\theta}+G(\theta) \\
T_{\text {motor }} & =K_{p}\left(\theta_{\text {des }}-\theta\right)+K_{d}\left(\dot{\theta}_{\text {des }}-\dot{\theta}\right)+G(\theta)
\end{aligned}
$$

Elimination of gravity terms and separating the $\theta$ terms on the left hand side would result in:

$$
\begin{aligned}
J_{e q} \ddot{\theta}+b_{e q} \dot{\theta}+K_{d} \dot{\theta}+K_{p} \theta & =K_{d} \dot{\theta}_{d e s}+K_{p} \theta_{\text {des }}+T_{\text {human }} \\
\frac{\theta(s)}{\theta_{\text {des }}(s)} & =\frac{K_{d} s+K p}{J_{e q} s^{2}+\left(b+K_{d}\right) s+K p} \\
\frac{\theta(s)}{T_{m}(s)} & =\frac{1}{J_{e q} s^{2}+\left(b+K_{d}\right) s+K p}
\end{aligned}
$$

The designed PID and gravity compensation controllers were used together to evaluate performance of the ankle platform.

\subsection{Performance evaluation}

A set of control tests were performed to evaluate and analyze the performance of the designed ankle actuation platform. First, the designed PID regulator was tested. The results are shown in Figure 4. The test showed that in all cases the platform reached the reference orientation within $300 \mathrm{~ms}$ which is sufficient for our study. To find suitable controller gains we first used the PD-regulator. Initially, the PD-gains were calculated from the platform's dynamics to achieve a critically damped response while assuming that the system does not have internal damping and gravity is perfectly compensated. Afterwards, the PID terms were further tuned to reduce the rise time and overshoot. The reason behind relatively slow response for the $5^{\circ}$ set-point and high steady state error for $15^{\circ}$ point is suspected to be the high static friction. To tackle this, a saturation was implemented on the error accumulation rate so that the increased integral 


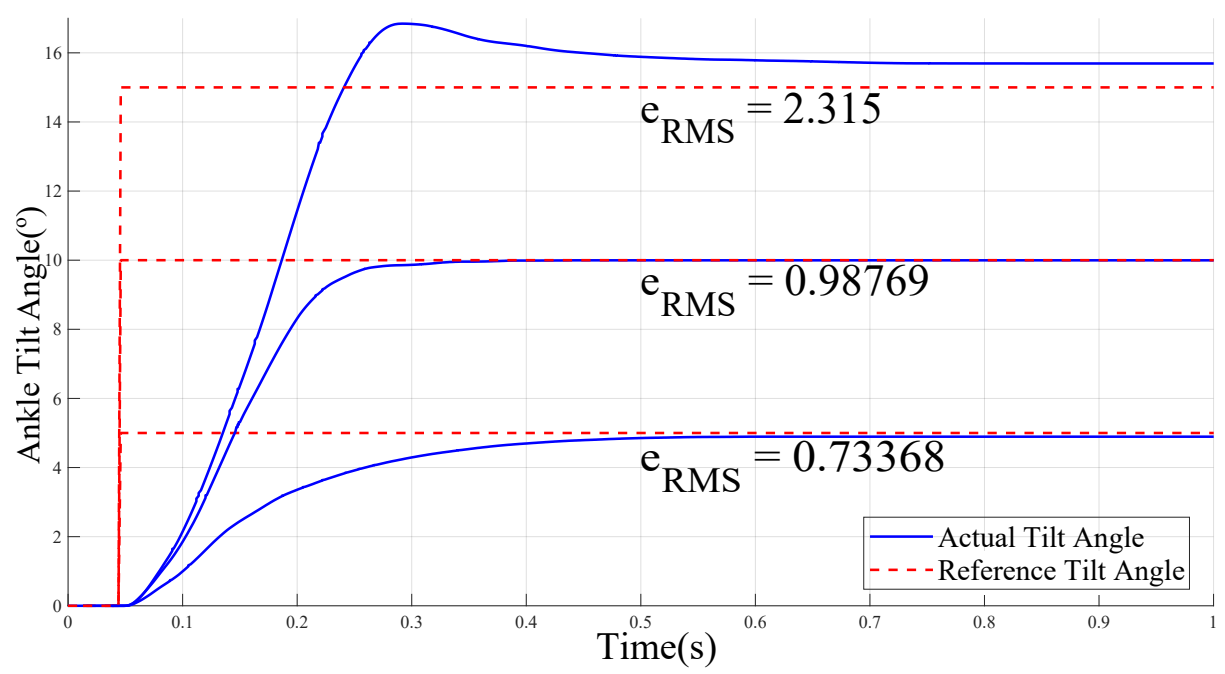

Fig. 4. Set-point reference tracking

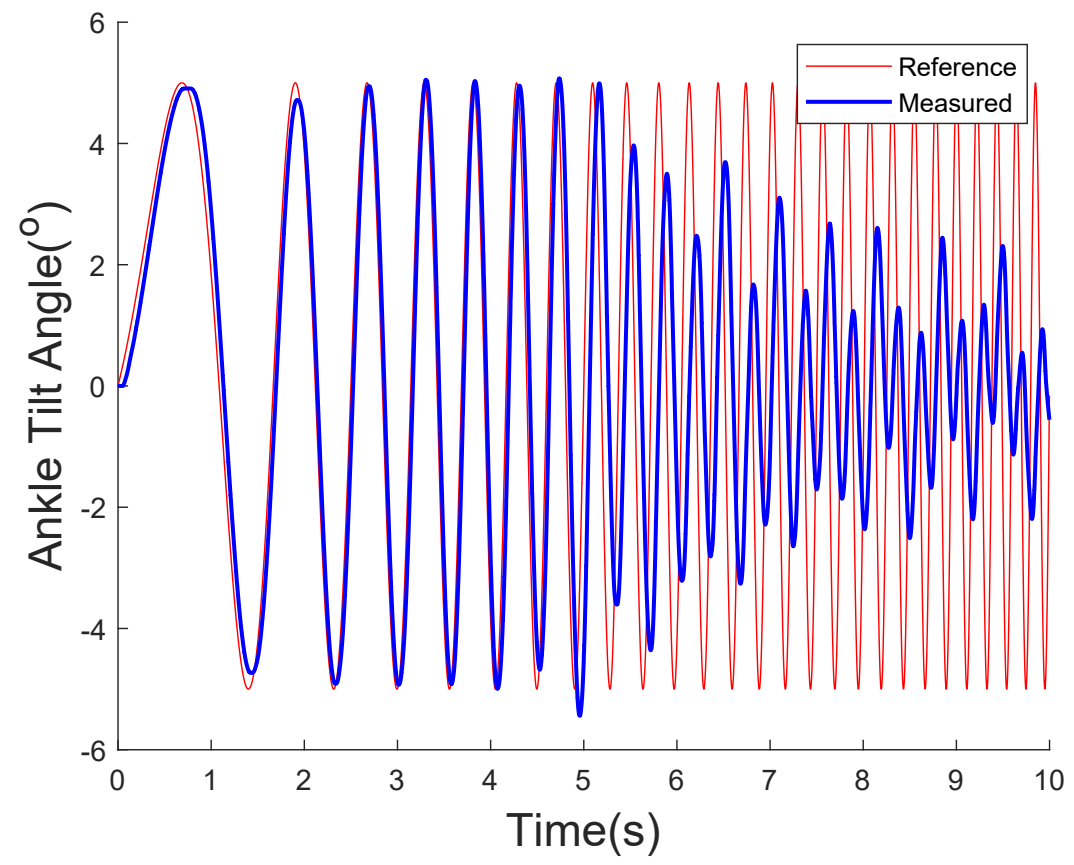

Fig. 5. Chirp reference tracking

gain would not be as effective in the increase of overshoot. The saturation limits 
the error at the maximum covered distance at each period by the device. A chirp reference tracking test was also performed to collect data on the position control bandwidth. The amplitude of the sinusoidal signal was set to be $5^{\circ}$ and the frequency of the signal was linearly increased from $0.2 \mathrm{~Hz}$ to $5 \mathrm{~Hz}$ over the course of 10 seconds. The results shown in Figure 5 suggest that the system can be used with good position tracking at frequencies up to $3 \mathrm{~Hz}$.

\subsection{Friction modeling}

This section elaborates on the modeling of the friction of the device which will be required for an accurate means of torque estimation with our impedance type device. As can be seen in the definitions of $J_{e q}$ and $b_{e q}$, the motor inertia and friction constitute a large part of the effective inertia at the ankle joint after multiplication with the gear ratios. For this reason, the motor was separately modeled for friction and inertia. Two tests were employed for this. Since there will not be multiple inertia or viscous friction terms, the apparent inertia and viscous friction coefficient on the output shaft of the motor will be referred to as $J$ and $b$.

The friction model we have used includes the components below:

- Static friction $\left(T_{s f}\right)$ : Friction threshold to overcome stiction.

- Coulomb friction $\left(T_{c f} \operatorname{sign}(\dot{\theta})\right)$ : Constant torque acting on the opposite direction of the movement.

- Stribeck effect $\left(T_{s f}=\left(T_{s f}-T_{c f}\right) e^{-\left(\dot{\theta} / w_{s}\right)^{\sigma}}\right)$ : Friction that is effective after the beginning of sliding and exponentially decays with increase in velocity.

- Viscous friction $\left(T_{v f}=b \dot{\theta}\right)$ : Torque acting on the opposite direction of the movement, proportional to the velocity.

Motor spin-down test: The motor is velocity controlled at different speeds and the torque input is suddenly cut. At high velocities only coulomb friction and viscous friction are effective, enabling an easier identification. The motor spins down until it stops. For speeds that are not close to zero, the equations are as follows.

$$
\begin{aligned}
& J \ddot{\theta}+b \dot{\theta}=T_{c f} \\
& J s \dot{\theta}-J \dot{\theta}(0) b \dot{\theta}(s)=T_{c f} / s \\
& \dot{\theta}(s)=\frac{J \dot{\theta}(0)}{J s+b}+\frac{T_{c f}}{s(J s+b)} \\
& \dot{\theta}=\left(\dot{\theta}(0)-\frac{T_{c f} t}{J}\right) * e^{-b / J}
\end{aligned}
$$

Figure 6 demonstrates data retrieved from spindown tests carried out at 4 distinct speeds. The characterization of the inertia, coulomb friction and viscous friction are performed using this data.

Open loop chirp torque test: The motor spin-down test only supplies the ratios, $T_{c f} / J$ and $\mathrm{b} / \mathrm{J}$, as there we primarily investigate the part where the 


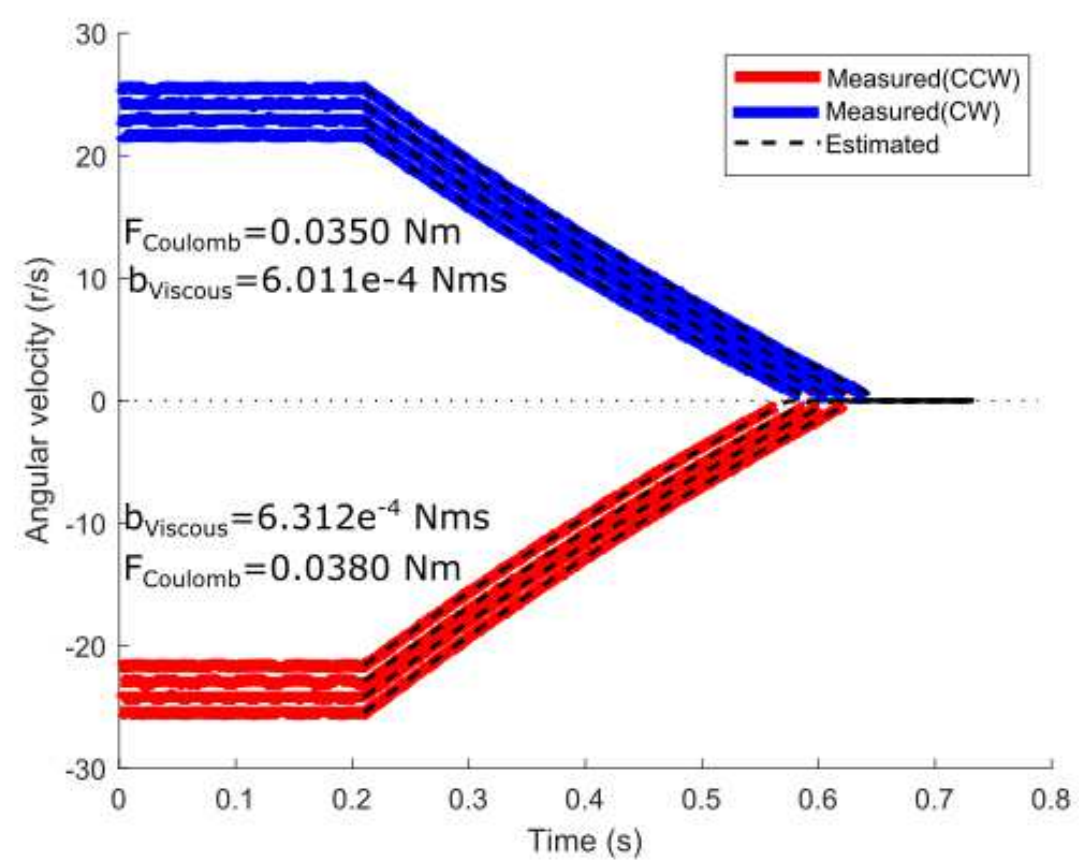

Fig. 6. Motor spin-down test starting from various velocities. The Coulomb and viscous friction are characterized separately for clockwise and counterclockwise cases.

input torque is zero. For this second test an open loop input signal is applied to the system and the output is recorded. Using the transfer functions between the angular velocity and the torque input along with the known ratios between $T_{c f}, \mathrm{~b}$ and $\mathrm{J}$ parameters reliable estimates could be achieved as demonstrated in Figure 7. Table 2 lists the resulting friction parameters estimation with the tests.

Table 2. Ankle platform characteristic parameters

\begin{tabular}{|l|l|l|l|}
\hline Parameter & Description & value & Unit \\
\hline \hline$J$ & inertia of the motor with & $7.540 \mathrm{e}^{-} 4$ & $\mathrm{~kg}-\mathrm{m}^{2}$ \\
& gear and pinion & & \\
\hline$b$ & viscous friction coefficient $(\mathrm{CW})$ & $6.312 \mathrm{e}^{-4}$ & $\mathrm{Nms}$ \\
& viscous friction coefficient $(\mathrm{CCW})$ & $6.011 \mathrm{e}^{-} 4$ & $\mathrm{Nm}$ \\
\hline$T_{c f}$ & Coulomb force $(\mathrm{CW})$ & 0.0350 & $\mathrm{Nm}$ \\
& Coulomb force $(\mathrm{CCW})$ & 0.0380 & $\mathrm{Nm}$ \\
\hline$w_{s}$ & Stribeck velocity & 3.2 & $\mathrm{r} / \mathrm{s}$ \\
\hline$T_{s f}$ & static friction & 0.045 & $\mathrm{Nm}$ \\
\hline
\end{tabular}




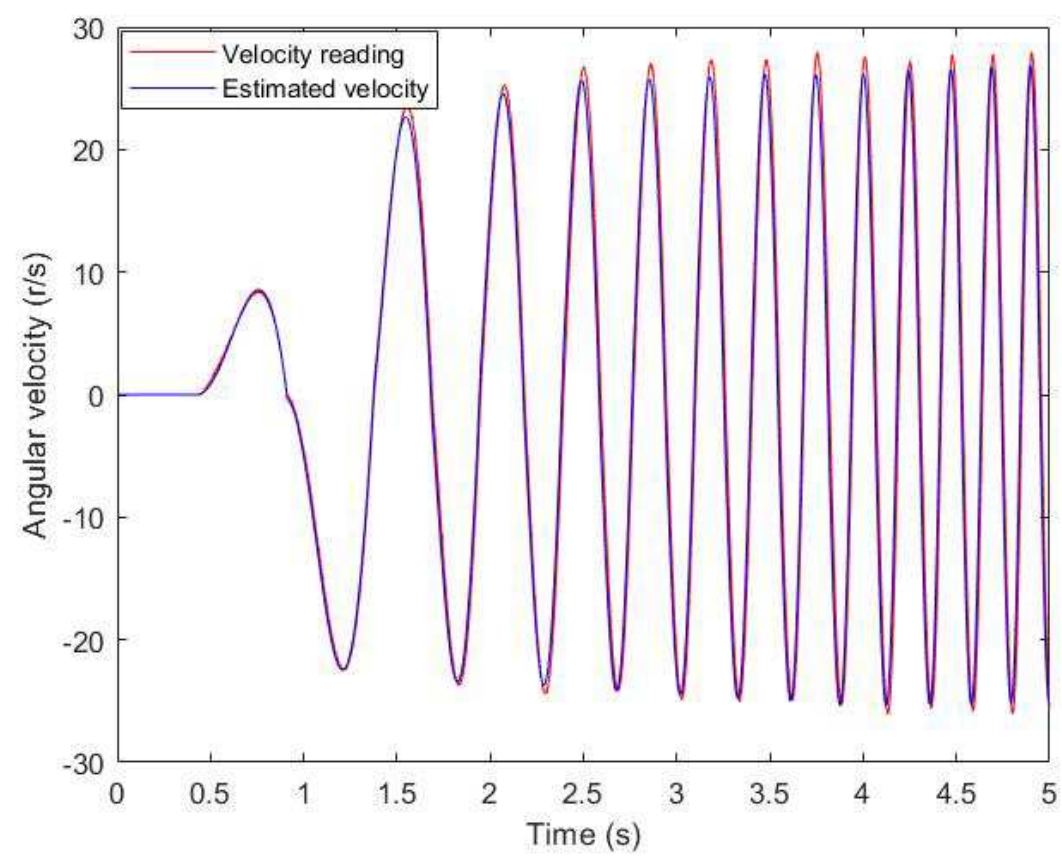

Fig. 7. Angular velocity vs. time graph generated with open loop torque sinusoidal input with linearly increasing frequency and amplitude

\section{Conclusion}

We have presented the design and preliminary modeling of a single DoF impedance type haptic interface for interactively actuating the ankle of a subject. The developed linear control with gravity and friction compensation allowed to achieve high performance movement response. The device was deliberately designed to be simple so that it is easier to augment it with further functionality useful for virtual reality and mobile robot teleroperation [5]. The system is currently being testing to investigate various aspects of self-motion perception [4] and spatial orientation in virtual reality.

\section{Acknowledments}

This work was funded by the UK EPSRC grant EP/R02572X/1 (NCNR). Ata Otaran was funded by Queen May University of London Ph.D. scholarship.

\section{References}

1. Buettner, D., Dalin, D., Wiesmeier, I.K., Maurer, C.: Virtual balancing for studying and training postural control. Frontiers in Neuroscience 11, 531 (2017) 
2. Dai, J.S., Zhao, T., Nester, C.: Sprained ankle physiotherapy based mechanism synthesis and stiffness analysis of a robotic rehabilitation device. Autonomous Robots 16(2), 207-218 (Mar 2004)

3. Erdogan, A., Celebi, B., Satici, A.C., Patoglu, V.: Assiston-ankle: a reconfigurable ankle exoskeleton with series-elastic actuation. Autonomous Robots 41(3), 743758 (Mar 2017)

4. Farkhatdinov, I., Ouarti, N., Hayward, V.: Vibrotactile inputs to the feet can modulate vection. 2013 World Haptics Conference (WHC) pp. 677-681 (2013)

5. Farkhatdinov, I., Ryu, J.H., Poduraev, J.: A user study of command strategies for mobile robot teleoperation. Intelligent Service Robotics 2(2), 95-104 (2009)

6. Girone, M., Burdea, G., Bouzit, M.: The rutgers ankle orthopedic rehabilitation interface. Proceedings of ASME Haptics Symposium 67, 305-312 (1999)

7. Goncalves, A.C.B.F., dos Santos, W.M., Consoni, L.J., Siqueira, A.A.G.: Serious games for assessment and rehabilitation of ankle movements. In: 2014 IEEE 3nd International Conference on Serious Games and Applications for Health (SeGAH). pp. 1-6 (May 2014)

8. Gupta, A., O'Malley, M.K.: Disturbance-observer-based force stimation for haptic feedback. vol. 133 (2010)

9. Liu, G., Gao, J., Yue, H., Zhang, X., Lu, G.: Design and kinematics analysis of parallel robots for ankle rehabilitation. In: 2006 IEEE/RSJ International Conference on Intelligent Robots and Systems. pp. 253-258 (Oct 2006)

10. Malosio, M., Negri, S.P., Pedrocchi, N., Vicentini, F., Caimmi, M., Molinari Tosatti, L.: A spherical parallel three degrees-of-freedom robot for ankle-foot neurorehabilitation. In: 2012 Annual International Conference of the IEEE Engineering in Medicine and Biology Society. pp. 3356-3359 (Aug 2012)

11. Roy, A., Krebs, H.I., Patterson, S.L., Judkins, T.N., Khanna, I., Forrester, L.W., Macko, R.M., Hogan, N.: Measurement of human ankle stiffness using the anklebot. In: 2007 IEEE 10th International Conference on Rehabilitation Robotics. pp. 356363 (June 2007)

12. Satici, A.C., Erdogan, A., Patoglu, V.: Design of a reconfigurable ankle rehabilitation robot and its use for the estimation of the ankle impedance. In: 2009 IEEE International Conference on Rehabilitation Robotics. pp. 257-264 (June 2009) 